\title{
Dynamical Casimir effect and minimal temperature in quantum thermodynamics
}

\author{
Giuliano Benenti ${ }^{1,2}$ and Giuliano Strini ${ }^{3}$ \\ ${ }^{1}$ Center for Nonlinear and Complex Systems, Università degli Studi dell'Insubria, via Valleggio 11, 22100 Como, Italy \\ ${ }^{2}$ Istituto Nazionale di Fisica Nucleare, Sezione di Milano, via Celoria 16, 20133 Milano, Italy \\ ${ }^{3}$ Department of Physics, University of Milan, via Celoria 16, 20133 Milano, Italy
}

\begin{abstract}
We study the fundamental limitations of cooling to absolute zero for a qubit, interacting with a single mode of the electromagnetic field. Our results show that the dynamical Casimir effect, which is unavoidable in any finite-time thermodynamic cycle, forbids the attainability of the absolute zero of temperature, even in the limit of an infinite number of cycles.
\end{abstract}

PACS numbers: 05.70.Ln, 07.20.Pe, 05.70.-a

Introduction. Due to recent progress of nanofabrication technology, quantum effects in small heat engines have become an increasingly important subject. Concepts from quantum thermodynamics [1] have been applied to investigate questions such as the optimization of quantum thermal machines [2], the fundamental dimensional limits to thermodynamic machines [3], and the minimum temperature achievable in nanoscopic chillers [4 11]. Cooling a system to the absolute zero of temperature $(T=0)$ is prohibited by Nernst's unattainability principle [12], also known as the dynamical formulation of the third law of thermodynamics. Such principle states that it is impossible by any procedure to reduce any system to $T=0$ in finite time. Nernst's principle has been recently challenged [8].

It this Letter, we investigate the unattainability principle in a minimal model: a qubit coupled to a single mode of the electromagnetic field, i.e. a harmonic oscillator. The oscillator is the working medium, shuttling heat from the qubit to a hot reservoir by means of a (quantum) Otto cycle. Although oversimplified, our model has several relevant features:

- The matter-field interaction is treated at the fundamental level of quantum electrodynamics.

- The equations of motion are solved by accurate numerical simulations, without resorting to the approximations necessarily involved in the master equations often used in the literature;

- We do not use the Rotating Wave Approximations (RWA), that neglects the effects of rapidly rotating terms in the equations of motion [13].

The latter point is particularly relevant where addressing the fundamental limits to cooling, since the terms beyond the RWA lead to the generation of photons from the vacuum due to time-dependent boundary conditions for the electromagnetic field. Such quantum vacuum amplification effect, known as the Dynamical Casimir Effect (DCE) [14 16], has been observed in recent experiments with superconducting circuits [17, 18], and also investigated in the context of Bose-Einstein condensates [19], in excition-polariton condensates [20], for multipartite entanglement generation in cavity networks 21] and for quantum communication protocols 22]. Here, we show that the DCE is a fundamental, purely quantum, limitation to cooling. We point out that the DCE is unavoidable in the context of finite-time thermodynamics [23] for cyclic quantum cooling machines, where the field's boundary conditions are effectively changed in time due to the switching on/off of the matter-field coupling [24]. In what follows, we show that, due to the $\mathrm{DCE}$, the $\mathrm{T}=0$ state of the qubit cannot be attained, even in the limit of infinite number of cooling cycles.

The model. We consider a reciprocating refrigerator, operating by means of a working medium [a single mode of the electromagnetic field, that is, a harmonic oscillator, with a time-dependent frequency $\omega(t)]$, shuttling heat from a cold finite-size "bath" (a single qubit) to a hot bath. The working medium undergoes a four-stroke Otto cycle:

- Isochore $\mathrm{A} \rightarrow \mathrm{B}$ : the working medium is in contact with the cold bath at temperature $T_{c}$; the work parameter, i.e. the oscillator frequency is maintained constant, $\omega(t)=\omega_{c}$.

- Adiabatic compression $\mathrm{B} \rightarrow \mathrm{C}$ : the frequency $\omega(t)$ of the working medium changes in time from $\omega_{c}$ to $\omega_{h}$.

- Isochore $\mathrm{C} \rightarrow \mathrm{D}$ : the working medium is in contact with the hot bath at temperature $T_{h}$; the oscillator frequency is constant, $\omega(t)=\omega_{h}$.

- Adiabatic expansion $\mathrm{D} \rightarrow \mathrm{A}$ : the frequency $\omega(t)$ changes from $\omega_{h}$ to $\omega_{c}$.

In order to elucidate the limitations imposed by the DCE on the lowest temperature that could be reached by a cooling protocol, we first consider the isochore stroke $\mathrm{A} \rightarrow \mathrm{B}$, with both the qubit and the oscillator prepared in their ground state. We wish to emphasize here that, even starting from these ideal conditions, due to the DCE both the oscillator and the qubit are excited, so that at the end of the isochore stroke the qubit is left in a state at a nonzero temperature. 
During the stroke $\mathrm{A} \rightarrow \mathrm{B}$ the qubit-oscillator interaction is described by the time-dependent Rabi Hamiltonian 13. (we set the reduced Planck's constant $\hbar=1$ ):

$$
\begin{gathered}
H(t)=H_{0}+H_{I}(t), \\
H_{0}=-\frac{1}{2} \omega_{a} \sigma_{z}+\omega\left(a^{\dagger} a+\frac{1}{2}\right), \\
H_{I}(t)=f(t)\left[g \sigma_{+}\left(a^{\dagger}+a\right)+g^{\star} \sigma_{-}\left(a^{\dagger}+a\right)\right],
\end{gathered}
$$

where $\sigma_{i}(i=x, y, z)$ are the Pauli matrices, $\sigma_{ \pm}=$ $\frac{1}{2}\left(\sigma_{x} \mp i \sigma_{y}\right)$ are the rising and lowering operators for the qubit: $\sigma_{+}|g\rangle=|e\rangle, \sigma_{+}|e\rangle=0, \sigma_{-}|g\rangle=0, \sigma_{-}|e\rangle=|g\rangle$; the operators $a^{\dagger}$ and $a$ for the field create and annihilate a photon: $a^{\dagger}|n\rangle=\sqrt{n+1}|n+1\rangle, a|n\rangle=\sqrt{n}|n-1\rangle$, $|n\rangle$ being the Fock state with $n$ photons. We first assume sudden switch on/off of the coupling: $f(t)=1$ for $0 \leq t \leq \tau, f(t)=0$ otherwise, with $\tau$ duration of the $\mathrm{A} \rightarrow \mathrm{B}$ stroke. For simplicity's sake, we consider the resonant case $\left(\omega=\omega_{a}\right)$ and the coupling strength $g \in \mathbb{R}$. The RWA (exact only in the limit $g \rightarrow 0$ ) is obtained when we neglect the term $\sigma_{+} a^{\dagger}$, which simultaneously excites the qubit and creates a photon, and $\sigma_{-} a$, which de-excites the qubit and annihilates a photon. In this limit, Hamiltonian (11) reduces to the Jaynes-Cummings Hamiltonian [13]. We set $\omega=1$, so that in the RWA the swap time needed to transfer an excitation from the qubit to the field or vice versa $(|e\rangle|0\rangle \leftrightarrow|g\rangle|1\rangle)$ is $\tau_{S}=\pi / 2 g$.

Results. If the qubit is prepared in its ground state, $\rho_{q}(0)=|g\rangle\langle g|$ and the oscillator is in the vacuum state, $\rho_{o}(0)=|0\rangle\langle 0|$, then, as we will discuss below, the qubit remains in a diagonal state in the basis of its eigenstates, $\rho_{q}(t)=p(t)|g\rangle\langle g|+[1-p(t)]| e\rangle\langle e|$. Therefore, the qubit's temperature reads as follows: $T(t)=$ $\omega / \ln \{p(t) /[1-p(t)]\}$ (we set the Boltzmann constant $k_{B}=1$ ). We found more convenient for visualization to plot, rather than the temperature, the $z$-coordinate of the Bloch vector [26] $\mathbf{r}(t)=[x(t), y(t), z(t)]$ of the state $\rho_{q}(t)=\frac{1}{2}(I+\mathbf{r}(t) \cdot \boldsymbol{\sigma})$, with $I$ identity operator and $\boldsymbol{\sigma}=\left(\sigma_{x}, \sigma_{y}, \sigma_{z}\right)$. In Fig. 11, we show $z(\tau)$ at the end of the isochore transformation $\mathrm{A} \rightarrow \mathrm{B}$, as a function of $\tau$ and of the qubit-oscillator coupling strength $g$. Within the RWA the initial tensor-product state $\rho_{q}(0) \otimes \rho_{o}(0)$ is the ground state of the overall qubit-oscillator system. Hence, $z(\tau)=1$, that is, the temperature $T(\tau)=0$ for any value of $\tau$. On the other hand, due to the DCE for any value of $g$ there exists a finite probability to generate photons and to excite the qubit, so that $z(\tau)<1$, namely the temperature $T(\tau)$ of the qubit is nonzero.

Since we are interested in the fundamental limitations to cooling imposed by the DCE, for the remaining part of the Otto cycle we ideally consider the most favorable conditions for cooling. That is, we assume that the adiabatic transformations can be performed without friction in a finite time by utilizing "shortcuts to adiabaticity" 27, 28]. Furthermore, we assume that the overall

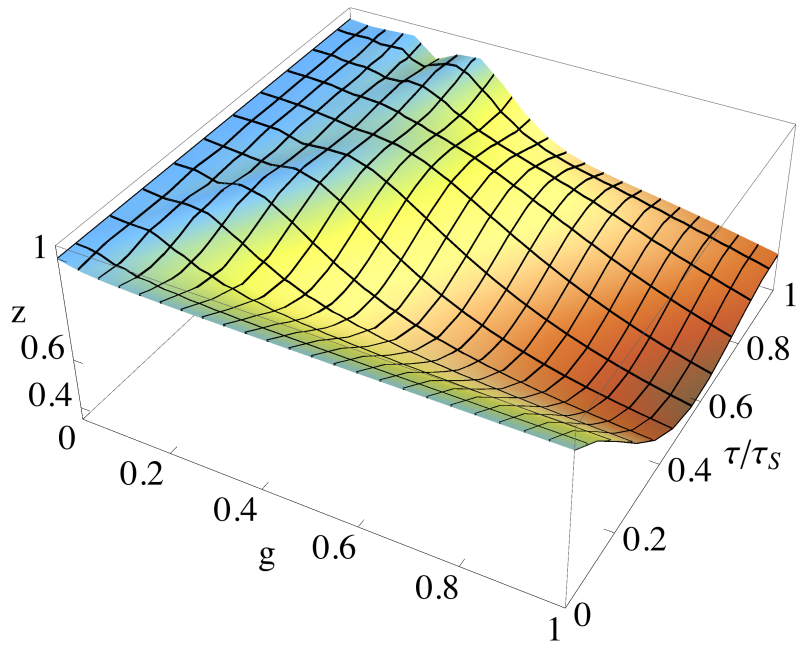

FIG. 1. (color online) Bloch coordinate $z$ of the qubit after the $\mathrm{A} \rightarrow \mathrm{B}$ isochore, as a function of the qubit-oscillator interaction time $\tau$ (in units of the swap time $\tau_{S}$ ) and the interaction strength $g$.

cycle is engineered in such a way that the oscillator is left in its vacuum state at the end of the cycle. While a careful treatment of the Otto cycle should be performed to evaluate the cooling power of a refrigerator [4, 5], our analysis, based on the most favorable instance, is sufficient to investigate the limitations set by the DCE to the lowest attainable temperature in finite-time thermodynamic cycles.

With the above assumptions, the state of the qubit after $n$ Otto cycles is given by

$$
\rho_{q, n}=\operatorname{Tr}_{o}\left[U\left(\rho_{q, n-1} \otimes|0\rangle\langle 0) \mid U^{\dagger}\right],\right.
$$

with $U$ the unitary time evolution operator describing the $\mathrm{A} \rightarrow \mathrm{B}$ evolution for the qubit and the oscillator and $\rho_{q, 0} \equiv \rho_{q}(0)$ initial state of the qubit. The quantum channel $\mathcal{E}$ mapping $\rho_{q, n-1}$ into $\rho_{q, n}$ can be conveniently described in the Fano-Bloch representation 22, 29 33]. If $\mathbf{r}_{n}=\left(x_{n}, y_{n}, z_{n}\right)$ denotes the Bloch vector of the state $\rho_{q, n}$, it follows from the linearity of quantum mechanics that $\mathbf{r}_{n-1}$ and $\mathbf{r}_{n}$ are connected through an affine map $\mathcal{M}$ :

$$
\left[\begin{array}{c}
\mathbf{r}_{n} \\
\hline 1
\end{array}\right]=\mathcal{M}\left[\frac{\mathbf{r}_{n-1}}{11}\right]=\left[\begin{array}{l|l}
\mathbf{M} & \mathbf{a} \\
\hline \mathbf{0}^{T} & 1
\end{array}\right]\left[\begin{array}{c}
\mathbf{r}_{n-1} \\
\hline 1
\end{array}\right],
$$

where $\mathbf{M}$ is a $3 \times 3$ real matrix, $\mathbf{r}_{n-1}, \mathbf{r}_{n}$ and a real column vectors of dimension 3 and $\mathbf{0}$ the null vector of the same dimension. The Fano-Bloch representation of quantum operations is also physically transparent since the Bloch vector directly provides the expectation values of polarization measurements. While in general an affine map for a qubit depends on twelve parameter [26], we found from the numerical simulation of the quantum map 
$\mathcal{E}$ the following structure of $\mathbf{M}$ and $\mathbf{a}$ :

$$
\mathbf{M}=\left(\begin{array}{ccc}
m_{x x} & m_{x y} & 0 \\
m_{y x} & m_{y y} & 0 \\
0 & 0 & m_{z z}
\end{array}\right), \quad \mathbf{a}=\left(\begin{array}{c}
0 \\
0 \\
a_{z}
\end{array}\right)
$$

It follows that, whatever the initial state $\rho_{q, 0}$ is, for $n \rightarrow \infty$ we have $x_{n}, y_{n} \rightarrow 0$ and $z_{n} \rightarrow a_{z} /\left(1-m_{z z}\right)$. Therefore the asymptotic state of the qubit, $\rho_{\infty}=$ $\frac{1}{2}\left(1+z_{\infty}\right)|g\rangle\left\langle g\left|+\frac{1}{2}\left(1-z_{\infty}\right)\right| e\right\rangle\langle e|$, is diagonal and we can readily derive the asymptotic temperature $T_{\infty}$. In the particular case in which the state is diagonal from the beginning, then, as observed above, it remains diagonal and the temperature $T_{n}$ can be computed as a function of the number $n$ of Otto cycles. Examples of the evolution of the Bloch ball coordinates $x_{n}, y_{n}$ and $z_{n}$ are shown in Fig. 2, for different initial states: the qubit's ground state $|g\rangle\langle g|$, the maximally mixed state $\frac{1}{2} I$, a thermal state $p|g\rangle\langle g|+(1-p)| e\rangle\langle e|$, with $p=0.6$, and the superposition state $\frac{1}{\sqrt{2}}(|g\rangle+|e\rangle)$. As expected, the $z$-coordinate converges to a limiting value depending only on the channel's control parameters (here, $g=0.5$ and $\tau=\pi / 2 g$ ) but not on the initial state of the qubit. For the superposition state we also show the asymptotic decay of $x$ and $y$ (in the other cases $x=y=0$ ).
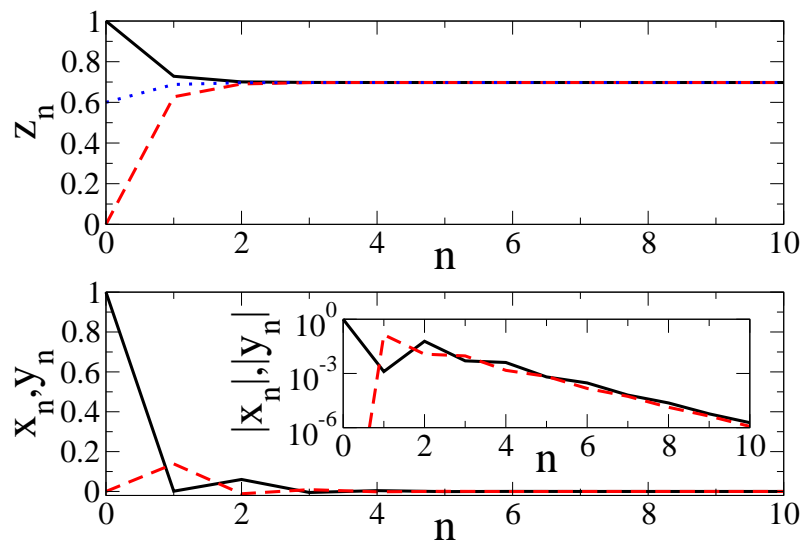

FIG. 2. (color online) Evolution of the Bloch ball coordinates as a function of the number of Otto cycles. Top: $z$-coordinate for the initial state of the qubit $|g\rangle\langle g|$ (black full curve), $\frac{1}{2} I$ (red dashed curve), $p|g\rangle\langle g|+(1-p)| e\rangle\langle e|$, with $p=0.6$ (blue dotted curve). In the bottom panel we show, for the state $\frac{1}{\sqrt{2}}(|g\rangle+|e\rangle)$ the $x$ - (black full curve) and the $y$-coordinate (red dashed curve). Note that for this state $z$ evolves exactly as for the maximally mixed input state. In the inset, for the same initial state, we show the exponential decay of $|x|$ and $|y|$.

In Fig. 3 we show the asymptotic value $z_{\infty}=$ $\lim _{n \rightarrow \infty} z_{n}$ as a function of the time $\tau$ and of the interaction strength $g$. It is clear from this plot that in our model only in the limit $g \rightarrow 0$ the temperature asymptotically vanishes. In the same figure, it might appear at first sight surprising that for a given $g$ and $\tau \rightarrow 0$ we have $z_{\infty} \rightarrow 0^{+}$, namely $T_{\infty} \rightarrow+\infty$. In this limit, we have $a_{z} \rightarrow 0^{+}$and $m_{z z} \rightarrow 1^{-}$, while the ratio $z_{\infty}=a_{z} /\left(1-m_{z z}\right) \rightarrow 0^{+}[35]$. Note that from the affine map (3) we have $z_{n}=m_{z z} z_{n-1}+a_{z}$, and therefore a value of $m_{z z}$ close to 1 implies a slow convergence to the asymptotic temperature.

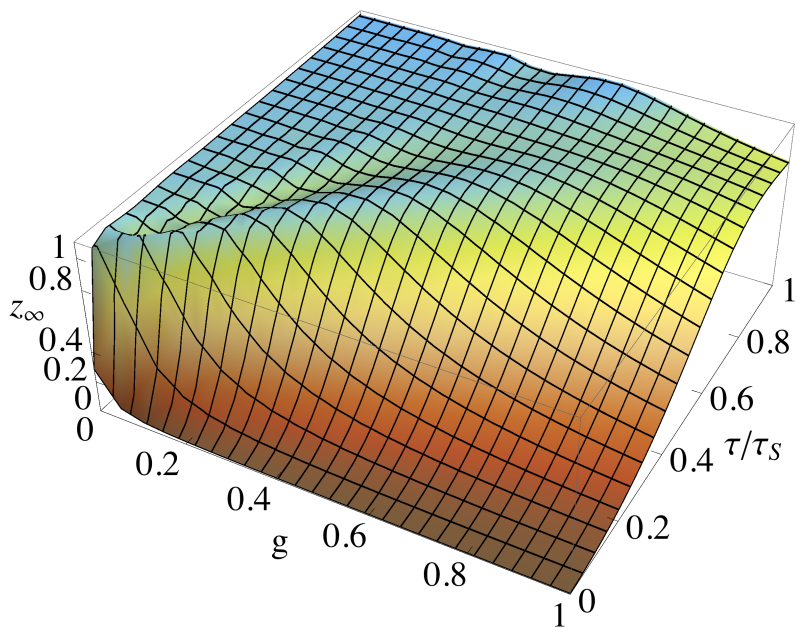

FIG. 3. (color online) Asymptotic value $z_{\infty}$ of the $z$ coordinate for the qubit, as a function of the time $\tau$ and the interaction strength $g$.

Finally, we investigate the effects of a smooth switch on/off of the interaction, by substituting in Eq. (1) the rectangular window so far considered with the Hamming window: $f(t)=\frac{1}{\alpha}[1-\cos (2 \pi t / \alpha \tau)]$ if $0 \leq t \leq \alpha \tau$, $f(t)=0$ otherwise. In particular, we consider the values $\alpha=1$, for which the interaction time (equal to $\tau$ ) is the same as for the rectangular window and the peak value (equal to $2 g$ ) is doubled, and $\alpha=2$, for which the interaction time $(2 \tau)$ is doubled and the peak value $(g)$ unchanged. In both cases, the area below the Hamming window is the same as for the rectangular window previously considered, so that such a window does not affect the cooling within the RWA, while significant differences appear in the ultra-strong coupling regime. In Fig. 4 we consider the time $\tau=\tau_{S}=\pi / 2 g$, so that within the RWA the asymptotic temperature $T_{\infty}=0$ [in the affine map (3), $m_{z z}=0$ and $a_{z}=1$ ]. More precisely, the qubit achieves the zero-temperature state in a single Otto cycle, since for this value of $\tau$ and for the oscillator prepared in the vacuum state, the qubit and the oscillator swap their state, leaving the qubit in its ground state. On the other hand, if the terms beyond the RWA are taken into account, the asymptotic temperature changes with respect to the rectangular window. The oscillations of $T_{\infty}$ with $g$ are smoothed; the temperature is raised for $\alpha=1$ and lowered for $\alpha=2$. This result can be understood from the adiabatic theorem: for $\alpha=2$ the interaction is switched on/off more gradually than for $\alpha=1$ and therefore, if we start from an eigenstate of the unperturbed 
Hamiltonian $H_{0}$, for $\alpha=2$ we end up, after the $A \rightarrow B$ isochore, on the same eigenstate with higher probability than for the case $\alpha=1$. In particular, if we start from the $T=0$ state, for the Hamming window such state is exactly preserved only in the limit $\alpha \rightarrow \infty$, that is, the qubit-field interaction time $\tau_{I}=\alpha \tau \rightarrow \infty$. In this limit the DCE vanishes since it is an effect due to the change of the boundary conditions for the field when it interacts with the qubit and such change becomes infinitely slow.

We should like to stress that the two limits of interaction time $\tau_{I} \rightarrow \infty$ and number of cycles $n \rightarrow \infty$ do not commute. If we let before $\tau_{I} \rightarrow \infty$, then we can achieve (in infinite time) the $T=0$ limit, as allowed from Nernst's principle. If on the other hand we set a large as desired but finite value of $\tau_{I}$ and then let $n \rightarrow \infty$, then the $T=0$ limit is not attained. This latter case is the one relevant for cyclic quantum chillers in finite-time thermodynamics.

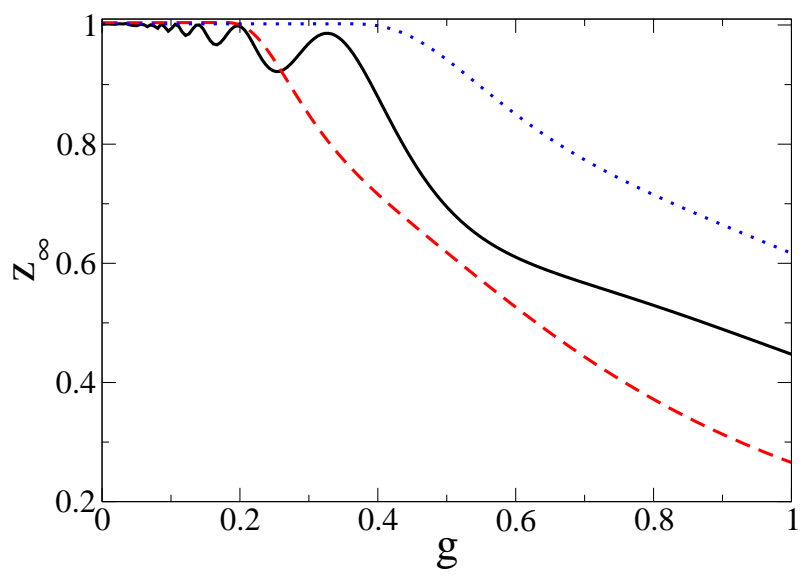

FIG. 4. (color online) Asymptotic value $z_{\infty}$ of the $z$ coordinate for the qubit, as a function of the interaction strength $g$, for the rectangular (black full curve) and the Hamming window for $\alpha=1$ (red dashed curve) or $\alpha=2$ (blue dotted curve). The interaction time $\tau=\tau_{S}=\pi / 2 g$.

Discussion and conclusions. In this paper, we have investigated the limitations imposed by the DCE on the minimal temperature achievable in a cooling cycle. We have considered a minimal model, where the purpose is to cool a single qubit and the working medium is a single mode of the electromagnetic field, undergoing a (quantum) Otto cycle. Since we are interested in the minimum attainable temperature, we have considered the most favorable instance in which the field is reset to its vacuum state at the beginning of each cycle.

Our model can be also interpreted as a collision model [36 42] of irreversible quantum dynamics. Such kind of models were used in the literature to analyze the process of thermalization of a system in contact with a bath. In our case, the qubit undergoes a sequence of identical collisions, described by the unitary evolution operator $U$ of Eq. (2), with the bath composed of an arbitrarily large number of oscillators initially in their ground state. Our results show that, due to the DCE, the qubit thermalizes to a nonzero temperature, different from the initial $T=0$ temperature of the bath of oscillators.

We emphasize that our results, though obtained for a very simple model, are exact, in that the coupled matterfield equations are numerically integrated, without (i) the RWA approximation and (ii) the master equation approximation often used in the literature. Since the DCE is a generic feature of finite-time quantum electrodynamics and therefore also of finite-time quantum thermodynamics, we conjecture that the unattainability of the zero temperature limit, even in the limit of infinite number of cycles, would remain valid for any cyclic quantum cooling machine. At any rate, our results call for a deeper understanding of the relevance of the dynamical Casimir effect in quantum thermodynamics.

G.B. acknowledges the support by MIUR-PRIN project "Collective quantum phenomena: From strongly correlated systems to quantum simulators".

[1] G. Gemma, M. Michel, and G. Mahler, G., Quantum thermodynamics (second Ed.) (Springer-Verlag, Berlin, 2009).

[2] G. Benenti, G. Casati, T. Prosen, and K. Saito, preprint arXiv:1311.4430

[3] N. Linden, S. Popescu, and P. Skrzypczyk, P. Phys. Rev. Lett. 105, 130401 (2010).

[4] Y. Rezek, P. Salamon, K. H. Hoffmann, and R. Kosloff, Europhys. Lett. 85, 30008 (2009).

[5] T. Feldmann, and R. Kosloff, Europhys. Lett. 89, 20004 (2010).

[6] A. Mari and J. Eisert, Phys. Rev. Lett. 108, 120602 (2012).

[7] B. Cleuren, B. Rutten, and C. Van den Broeck, Phys. Rev. Lett. 108, 120603 (2012).

[8] M. Kolář, D. Gelbwaser-Klimovsky, R. Alicki, and G. Kurizki, Phys. Rev. Lett. 109, 090601 (2012).

[9] A. Levy and R. Kosloff, Phys. Rev. Lett. 108, 070604 (2012).

[10] A. Levy, R. Alicki, and R. Kosloff, Phys. Rev. E 85, 061126 (2012).

[11] E. Torrontegui and R. Kosloff, Phys. Rev. E 88, 032103 (2013).

[12] W. Nernst, The new heat theorem, its foundations in theory and experiment (Dutton, New York, 1926).

[13] P. Meystre and M. Sargent III, Elements of quantum optics (4th Ed.) (Springer-Verlag, Berlin, 2007).

[14] G. T. Moore, J. Math. Phys. (N.Y.) 11, 2679 (1970).

[15] V. V. Dodonov, Phys. Scripta 82, 038105 (2010).

[16] P. D. Nation, J. R. Johansson, M. P. Blencowe, and F. Nori, Rev. Mod. Phys. 84, 1 (2012).

[17] C. M. Wilson, G. Johansson, A. Pourkabirian, M. Simoen, J. R. Johansson, T. Duty, F. Nori, and P. Delsing, Nature (London) 479, 376 (2011).

[18] P. Lähteenmäki, G. S. Paraoanu, J. Hassel, and P. J. 
Hakonen, PNAS 110, 4234 (2013).

[19] J.-C. Jaskula, G. B. Partridge, M. Bonneau, R. Lopes, J. Ruaudel, D. Boiron, and C. I. Westbrook, Phys. Rev. Lett. 109, 220401 (2012).

[20] S. Koghee and M. Wouters, Phys. Rev. Lett. 112, 036406 (2014).

[21] S. Felicetti, M. Sanz, L. Lamata, G. Romero, G. Johansson, P. Delsing, and E. Solano, Phys. Rev. Lett. 113, 093602 (2014).

[22] G. Benenti, A. D'Arrigo, S. Siccardi, and G. Strini, Phys. Rev. A 90, 052313 (2014).

[23] B. Andresen, Angew. Chem. Int. Ed. 50, 2690 (2011).

[24] We will consider a model without moving boundaries, but with a time-dependent matter-field coupling $g(t)$. Since the coupling constant $g \propto 1 / \sqrt{V}$, with $V$ the quantization volume for the field, changing $g$ in time is equivalent to changing the volume $V$, see Ref. 25].

[25] G. Benenti, S. Siccardi, and G. Strini, Eur. Phys. J. D 68, 139 (2014).

[26] G. Benenti, G. Casati, and G. Strini, Principles of Quantum Computation and Information, Vol. I: Basic concepts (World Scientific, Singapore, 2004); Vol. II: Basic tools and special topics (World Scientific, Singapore, 2007).

[27] E. Torrontegui, S. Ibánẽz, S. Martínez-Garaot, M. Modugno, A. del Campo, D. Guéry-Odelin, A. Ruschhaupt, X. Chen, and J. G. Muga, Adv. At. Mol. Opt. Phys. 62, 117 (2013).

[28] A. del Campo, J. Goold, and M. Paternostro, Sci. Rep. 4, 6208 (2014).

[29] U. Fano, Rev. Mod. Phys. 29, 74 (1957); ibid. 55, 855 (1983).
[30] F. T. Hioe and J. H. Eberly, Phys. Rev. Lett. 47, 838 (1981).

[31] J. Schlienz and G. Mahler, Phys. Rev. A 52, 4396 (1995).

[32] G. Benenti and G. Strini, Phys. Rev. A 80, 022318 (2009).

[33] G. Benenti and G. Strini, J. Phys. B: At. Mol. Opt. Phys. 43, 215508 (2010).

[34] G. Benenti, S. Felloni, and G. Strini, Eur. Phys. J. D 38, 389 (2006).

[35] More precisely, from numerical data as well as from a second order (in the interaction Hamiltonian $H_{I}$ ) perturbative treatment of finite-time quantum electrodynamics, outlined in Ref. [25], we find that

$$
z_{\infty}=\frac{1}{6}(\omega \tau)^{2}\left[1+\frac{9 g^{2}}{4 \omega^{2}}\right] .
$$

[36] V. Scarani, M. Ziman, P. Štelmachovič, N. Gisin, and V. Bužek, Phys. Rev. Lett. 88, 097905 (2002).

[37] M. Ziman, P. Štelmachovič, V. Bužek, M. Hillery, V. Scarani, and N. Gisin, Phys. Rev. A 65, 042105, (2002).

[38] M. Ziman and V. Bužek, Phys. Rev. A 72, 022110, (2005).

[39] M. Koniorczyk, A. Varga, P. Rapčan, and V. Bužek, Phys.Rev A 77, 052106 (2008).

[40] G. Gennaro, G. Benenti, and G. M. Palma, Phys. Rev. A 79, 022105 (2009).

[41] V. Giovannetti and G. M. Palma, Phys. Rev. Lett. 108, 040401 (2012).

[42] F. Ciccarello, G. M. Palma, and V. Giovannetti, Phys. Rev. A 87, 040103(R) (2013). 